\title{
Retinal Dystrophy and Optic Nerve Pathology in the Mouse Model of Mucolipidosis IV
}

Yulia Grishchuk, ${ }^{*}$ Katherine G. Stember, ${ }^{*}$ Aya Matsunaga, ${ }^{*}$ Ana M. Olivares, ${ }^{\dagger}$ Nelly M. Cruz, ${ }^{\dagger}$ Victoria E. King, ${ }^{*}$ Daniel M. Humphrey, ${ }^{*}$ Shirley L. Wang, ${ }^{*}$ Alona Muzikansky, ${ }^{\ddagger}$ Rebecca A. Betensky, ${ }^{\S}$ Wallace B. Thoreson, ${ }^{\top}$ Neena Haider, ${ }^{\dagger}$ and Susan A. Slaugenhaupt*

\begin{abstract}
From the Department of Neurology,* Center for Human Genetic Research, Harvard Medical School, and the Massachusetts General Hospital Biostatistics Center, ${ }^{\ddagger}$ Massachusetts General Hospital, Boston, Massachusetts; the Schepens Eye Research Institute, ${ }^{\dagger}$ Massachusetts Eye and Ear Infirmary, Boston, Massachusetts; the Department of Biostatistics, ${ }^{\S}$ Harvard School of Public Health, Boston, Massachusetts; and the Department of Ophthalmology \& Visual Sciences, "University of Nebraska Medical Center, Omaha, Nebraska
\end{abstract}

Accepted for publication September 10, 2015.

Address correspondence to Yulia Grishchuk, Ph.D., Center for Human Genetic Research, Harvard Medical School and Massachusetts General Hospital, 185 Cambridge St, Boston, MA 02114. E-mail: ygrishchuk@mgh.harvard.edu.
Mucolipidosis IV is a debilitating developmental lysosomal storage disorder characterized by severe neuromotor retardation and progressive loss of vision, leading to blindness by the second decade of life. Mucolipidosis IV is caused by loss-of-function mutations in the MCOLN1 gene, which encodes the transient receptor potential channel protein mucolipin-1. Ophthalmic pathology in patients includes corneal haze and progressive retinal and optic nerve atrophy. Herein, we report ocular pathology in Mcoln1 $1^{-/}$mouse, a good phenotypic model of the disease. Early, but non-progressive, thinning of the photoreceptor layer, reduced levels of rhodopsin, disrupted rod outer segments, and widespread accumulation of the typical storage inclusion bodies were the major histological findings in the $M$ coln $1^{-/-}$retina. Electroretinograms showed significantly decreased functional response (scotopic a- and b-wave amplitudes) in the Mcoln $1^{-/-}$ mice. At the ultrastructural level, we observed formation of axonal spheroids and decreased density of axons in the optic nerve of the aged (6-month-old) Mcoln $1^{-/-}$mice, which indicates progressive axonal degeneration. Our data suggest that mucolipin-1 plays a role in postnatal development of photoreceptors and provides a set of outcome measures that can be used for ocular therapy development for mucolipidosis IV. (Am J Pathol 2016, 186: 199-209; http://dx.doi.org/10.1016/j.ajpath.2015.09.017)
Mucolipidosis type IV (MLIV) is an autosomal recessive disease characterized by severe psychomotor retardation and visual loss. MLIV is classified as a lysosomal storage disease because of abnormal accumulation of storage material in lysosomes of all cells and tissues of the body. ${ }^{1}$ Corneal clouding because of accumulation of lysosomal storage is an early pathological hallmark of the disease that, when present with developmental delay in infanthood, is highly suggestive of MLIV.

Ophthalmic manifestations in patients generally have a progressive course and, in addition to corneal clouding, include optic nerve atrophy and outer retinal degeneration. $^{2-4}$ In most of the patients, MLIV leads to blindness in the second decade of life. ${ }^{5}$

Mutations in MCOLN1, which encodes the transient receptor potential cation channel TRPML1 (alias mucolipin-1) cause the disease. ${ }^{6-9}$ More than 20 mutations in MCOLN1 have been identified to date. ${ }^{5}$ More than $75 \%$ of known MLIV patients are Ashkenazi Jewish, and the two founder mutations, present in $95 \%$ of Ashkenazi Jewish patients,

\footnotetext{
Supported by an ML4 Foundation grant (Y.G. and S.A.S.), a Research to Prevent Blindness Senior Scientific Investigator Award (W.B.T.), NIH National Eye Institute core grant (P30EY003790), NIH Award 8UL1TR000170-05, and financial contributions from Harvard University and its affiliated academic health care centers. Statistical analysis was conducted with support from Harvard NeuroDiscovery Center and Harvard Catalyst (National Center for Research Resources and the National Center for Advancing Translational Sciences, NIH Award 8UL1TR000170-05, and financial contributions from Harvard University and its affiliated academic health care centers). SERI electron microscopy core is supported through an NIH NEI Core grant (P30EY003790).

The content is solely the responsibility of the authors and does not necessarily represent the official views of Harvard Catalyst, Harvard University, and its affiliated academic health care centers, or the NIH.

Disclosures: None declared.
} 
result in complete loss of mRNA and protein. ${ }^{10}$ MLIV is a rare disease with carrier frequency of 1:100 in Ashkenazi Jewish and 1:10,000 in the general population. Many patients with MLIV remain undiagnosed or are misdiagnosed with cerebral palsy. Thus, bringing awareness of this disease to pediatric ophthalmologists and neurologists is important to improve diagnosis as new therapies are developed.

Mucolipin-1 has six transmembrane domains and is permeable to $\mathrm{Ca}^{2+}, \mathrm{Na}^{+}, \mathrm{K}^{+}, \mathrm{Fe}^{2+}, \mathrm{Mn}^{2+}$, and $\mathrm{Zn}^{2+} \cdot{ }^{11-13}$ Its channel activity is regulated by both calcium concentration and $\mathrm{pH}$, and mucolipin-1 has been shown to have lipase activity. ${ }^{14}$ Previous studies by our group and others showed mucolipin-1 localization to the late endosomes and lysosomes. ${ }^{15-18}$ The transient receptor potential channel protein mucolipin-1 is required for transport of lipids from the late endosomes-lysosomes to the trans-Golgi compartment, ${ }^{19,20}$ $\mathrm{Ca}^{2+}$-dependent late endosome-lysosome fission-fusion events, ${ }^{20}$ reformation of lysosomes from endosome-lysosome hybrids ${ }^{18,21}$ and autolysosomes, ${ }^{22,23}$ and lysosomal exocytosis. ${ }^{24,25}$ Mucolipin-1 is strongly expressed in the mouse retina, with the highest mRNA levels in the outer plexiform layer and outer nuclear layer. ${ }^{26}$

The Mcoln1 knockout (KO) mouse model recapitulates the main features of the human disease, and is a good phenotypic platform for investigating MLIV disease mechanisms. ${ }^{27-30}$ At the ultrastructural level, typical MLIV storage inclusions have been found in the brain during embryonic development. ${ }^{31}$ Histochemical analysis in the young adult ( 2 months of age) Mcoln $1^{-1-}$ mice has shown pronounced glial activation, reduced myelination, and no neuronal loss in the cerebrum in the regions most affected by gliosis. ${ }^{28}$

In this study, we used $M \operatorname{coln} 1^{-1-}$ mice to characterize the consequences of mucolipin-1 loss on retinal morphology, optic nerve myelination, and visual function in the course of the disease. Major manifestations of ophthalmic pathology in $M \operatorname{coln} 1^{-1-}$ mice were as follows: non-progressive thinning of the photoreceptor layer; profound accumulation of storage inclusions throughout the retina and in the optic nerve, including formation of large axonal spheroids; axonal degeneration in the optic nerve in older mice; hypertrophied lysosomes in photoreceptors and other retinal cells; and reduced visual function.

\section{Materials and Methods}

\section{Animals}

Mcoln1 KO mice were maintained and genotyped as described previously. ${ }^{30}$ The $M$ coln $1^{+/-}$breeders for this study were obtained by backcrossing onto a C57B1/6J background. Initial characterization of the $M c o l n 1^{-/-}$mouse ${ }^{30}$ reported severely disorganized retina. This more severe phenotype (compared with the data presented in this study) is likely because of the presence of the rd8 mutation ${ }^{32}$ in the genetic background of the Mcolnl $^{-/-}$mouse colony maintained on C57B1/6N background, which exacerbates retinal phenotype. For the purpose of the present study, the rd8 mutation was eliminated from the $M \operatorname{coln} 1^{-1-}$ colony by backcrossing to C57B1/6J mice (Jackson Biolabs, Bar Harbor, ME). Mcoln $1^{+/+}$littermates were used as controls for all experiments. All experiments were performed according to the US NIH guidelines and approved by the Massachusetts General Hospital and the Schepens Eye Research Institutional Animal Care and Use Committees.

\section{Histochemistry}

Eyes were collected, cauterized, fixed in either $4 \%$ paraformaldehyde (PFA) in $1 \times$ phosphate-buffered saline or methanol/acetic acid (3:1, vol:vol) overnight at $4^{\circ} \mathrm{C}$, and embedded in either Tissue-Tek OCT or paraffin. Paraffinor OCT-embedded frozen tissue was divided into serial sections (5 $\mu \mathrm{m}$ thick).

For morphometric analysis, methanol-acetate-fixed mice ( $n=4$ [wild type (WT)] and $n=5[\mathrm{KO}]$ at 1 month; $n=3$ [WT] and $n=5[\mathrm{KO}]$ at 2 months; $n=4$ [WT] and $n=4$ $[\mathrm{KO}]$ at 6 months) were stained with hematoxylin and eosin, as described previously. ${ }^{33}$ Images were obtained on an Olympus BX51 phase-contrast light microscope equipped with an Olympus ColorQ5 camera (Olympus America Inc., Center Valley, PA). Fiji win64 software (NIH, Bethesda, $\mathrm{MD}$ ) was used for image processing and analysis. For the outer nuclear layer measurements, the number of photoreceptor rows was estimated at the thickest part of the retina and $100 \mu \mathrm{m}$ from this point to the right and to the left. The average value of these three measurements was used as number of photoreceptor rows per section. For outer segment thickness measurements, the scale was calibrated in the Fiji software and the line tool was used to draw a perpendicular line from the outer nuclear layer to the retinal pigment epithelium. The length of this line corresponded to the thickness of the outer segments. Three measurements were taken per section: in the center of the section, $100 \mu \mathrm{m}$ to the left from the center, and $100 \mu \mathrm{m}$ to the right from the center. In total, six adjacent sections were analyzed in each mouse, within three to five mice per genotype for each time point. Two-way analysis of variance (GraphPad Prism software version 5; GraphPad, La Jolla, CA) was used to analyze the effect of genotype and age on the thickness of the outer nuclear layer and photoreceptor segments.

Immunofluorescence staining was performed as described previously. ${ }^{34}$ In brief, paraffin sections were deparaffinized by incubation in xylene, $100 \%, 95 \%$, and $70 \%$ ethanol solutions. PFA-fixed sections were microwaved at maximal power for 60 seconds in $10 \mathrm{mmol} / \mathrm{L}$ citric acid buffer (pH 6.0) for antigen retrieval. Eye sections were blocked with horse normal serum (1:50 in phosphate-buffered saline) at room temperature for 1 hour, and the same blocking buffer was used for antibody dilution. The following primary antibodies were used on methanol-acetate-fixed/ paraffin-embedded sections: rhodopsin (1:500, mouse monoclonal, MAB5356; Chemicon, Temecula, CA); tubulin 
III (1:400, rabbit, T2200; Sigma, St. Louis, MO); parvalbumin (1:200, rabbit, AB11427; Abcam, Cambridge, MA); S-cone opsin (1:200; rabbit, AB5407; Millipore, Billerica, MA); M-cone opsin (1:200, rabbit, AB5405; Millipore); Lamp1 (1:500, rat, number 553792; BD Biosciences, San Jose, CA); glial fibrillary acidic protein (GFAP; 1:1000, mouse, number 3670; Cell Signaling, Danvers, MA), ionized calcium-binding adapter molecule 1 (Iba1) (1:500, rabbit, number 016-20001; Wako, Richmond, VA), p62/ sequestosome 1 (1:500, rabbit, PW9860; Enzo Life Sciences, Farmingdale, NY), calbindin (1:1000, rabbit, AB1778; Millipore), and protein kinase C- $\alpha$ (1:500, mouse, SC8393; Santa Cruz Biotechnology, Dallas, TX) were used on PFA-fixed/Tissue-Tek OCT-embedded cryosections. The following secondary antibodies were applied (all at 1:400 dilution): donkey-anti-rat AF546, donkey-anti-rabbit AF555, goat-anti-rabbit AF633, goat-anti-rabbit AF488, and goat-anti-mouse AF633 (all from Molecular Probes, Invitrogen, Waltham, MA). To detect cones, biotinylated peanut agglutinin (1:200, B-1075; Vector Laboratories, Burlingame, CA) was used on methanol-acetate-fixed/ paraffin-embedded sections after blocking using the same immunohistochemical protocol, followed by incubation with streptavidin Alexa Fluor 555 conjugate (1:1000, S32355; Molecular Probes).

To detect apoptotic cells in the retina, a terminal deoxynucleotidyl transferase-mediated dUTP nick-end labeling (TUNEL) assay was performed using the In Situ Cell Death Detection Kit with Fluorescein (number 11684795 910; Roche, Mannheim, Germany). PFA-fixed/paraffinembedded sections were deparaffinized in xylene and dehydrated in the series of ethanols $(100 \%, 95 \%, 70 \%$, $2 \times$, for 5 minutes each time). For antigen retrieval, slides were irradiated in $0.1 \mathrm{~mol} / \mathrm{L}$ citrate buffer, $\mathrm{pH} 6.0$, in a microwave at high power for 2 minutes, cooled to room temperature, and blocked for 1 hour in $0.1 \mathrm{~mol} / \mathrm{L}$ Tris- $\mathrm{HCl}$ containing 3\% bovine serum albumin and $20 \%$ normal horse serum. After blocking, slides were incubated with the TUNEL reaction mix for 1 hour at $37^{\circ} \mathrm{C}$ in a humidified atmosphere in the dark. After incubation, sections were washed in phosphate-buffered saline $(3 \times, 5$ minutes each) and counterstained with DAPI. Fluorescent microscopy was performed using a Leica DMI6000 live cell imaging fluorescent microscope (Leica Microsystems, Wetzlar, Germany). The number of apoptotic cells per retinal section was counted on four sections per animal, and the mean values were compared in groups of three to five animals per genotype at 1, 2, and 6 months of age. A $t$-test was used to compare the average number of apoptotic cells per retina between Mcoln1 ${ }^{-1-}$ and WT littermates at each time point.

\section{ERG}

For electroretinographic (ERG) recordings, 1-month-old $M \operatorname{coln} 1^{-1-}(n=13)$ and Mcoln $1^{+/+}(n=14)$ mice were anesthetized with an i.p. injection of a mixture of $1 \mathrm{mg} / \mathrm{mL}$ ketamine and $0.4 \mathrm{mg} / \mathrm{mL}$ xylazine in a saline carrier $(10 \mathrm{mg} / \mathrm{g}$ of body weight), and mouse eyes were dilated with $1 \%$ tropicamide and $2.5 \%$ phenylephrine hydrochloride applied topically. ERGs were performed using Espion Visual Electrophysiology System (Diagnoysis, Littleton, MA), as described previously, ${ }^{34,35}$ to record both the dark- and the light-adapted responses. For scotopic recordings, mice were dark adapted for at least 6 hours and then anesthetized before recording. Dark-adapted responses were recorded to short wavelength $(\operatorname{lmax}=470 \mathrm{~nm}$; Wratten 47A filter; Kodak, Rochester, NY) flashes of light over a 4.0-log unit range of intensities (0.3-log unit steps) up to the maximum allowable by the photic stimulator. Light-adapted responses were obtained with white flashes ( 0.3 step) on the rod-saturating background after 10 minutes of exposure to the background light to allow complete light adaptation. Signal processing was performed using EMWIN software version 7.1.2 (LKC Technologies, Inc., Gaithersburg, MD). Signals were sampled every 0.8 milliseconds over a response window of 200 milliseconds. Responses were averaged for each stimulus condition, with up to 50 records for the weakest signals.

\section{Fundus and Optical Coherence Tomography Imaging}

For fundus and optical coherence tomography examination, mice were anesthetized with an i.p. injection of a mix of $1 \mathrm{mg} / \mathrm{mL}$ ketamine and $0.4 \mathrm{mg} / \mathrm{mL}$ xylazine in a saline carrier $(10 \mathrm{mg} / \mathrm{g}$ of body weight), and eyes were dilated with $1 \%$ tropicamide and $2.5 \%$ phenylephrine hydrochloride applied topically. A fundus examination was performed as described previously, ${ }^{36,37}$ and spectral domain-optical coherence tomography images were taken using the Micron III imaging system (Phoenix Research Labs, Pleasanton, CA).

\section{Western Blot Analysis}

For immunoblotting, eyes $(n=3$ per genotype per time point) were homogenized in radioimmunoprecipitation assay buffer (Boston BioProducts, Ashland, MA) supplemented with $0.1 \mathrm{mmol} / \mathrm{L}$ EDTA and a protease inhibitor cocktail (11873580001; Roche). Homogenates were centrifuged at $2100 \times g$ for 10 minutes at $4^{\circ} \mathrm{C}$, supernatants were collected, and protein concentration was determined using the Pierce BSA Protein Assay kit (ThermoFisher Scientific, Waltham, MA). Proteins (20 to $40 \mu \mathrm{g}$ ) were separated by SDS-PAGE on $12 \%$ and $4 \%$ to $12 \%$ Tris-acetate gels, in 2-( $N$-morpholino)ethanesulfonic acid or 3-( $N$-morpholino) propanesulfonic acid buffer (Invitrogen) and transferred onto a nitrocellulose membrane (ThermoFisher Scientific) for immunodetection. The following primary antibodies were used: anti-rhodopsin (MAB5356, 1:1000, mouse monoclonal; Chemicon), anti-M-cone opsin (AB5405, 1:1000, rabbit polyclonal) and anti-S-cone opsin (AB5407, 1:1000, rabbit polyclonal) (both from Millipore), anti-Iba1 
A

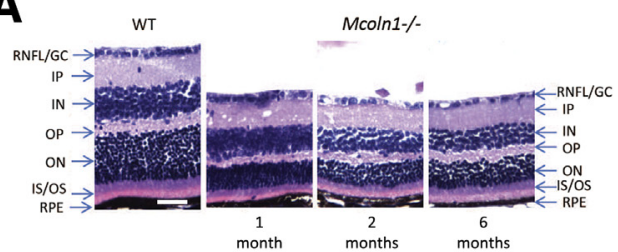

C

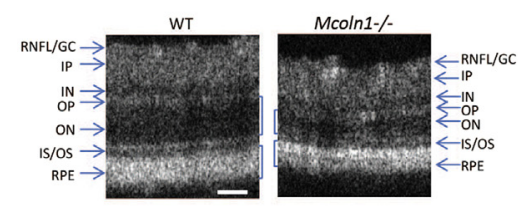

E

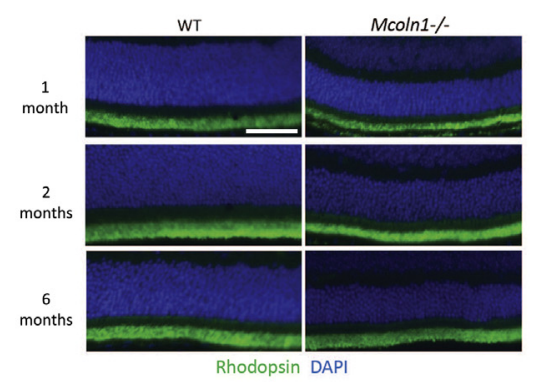

G

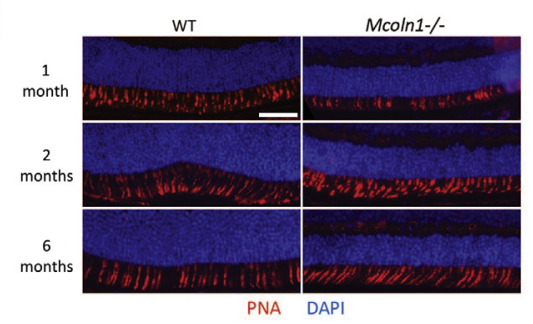

B

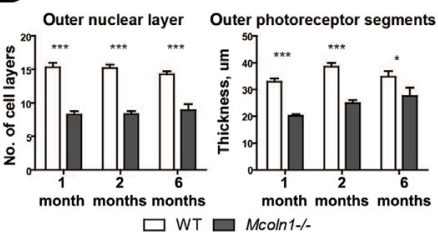

D

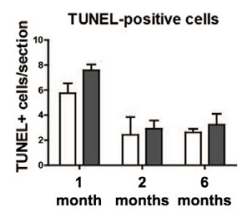

\section{$\mathbf{F}$}
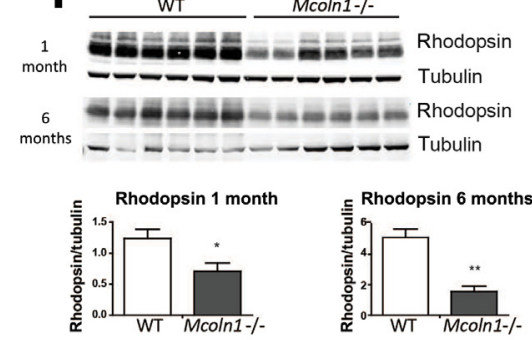

H

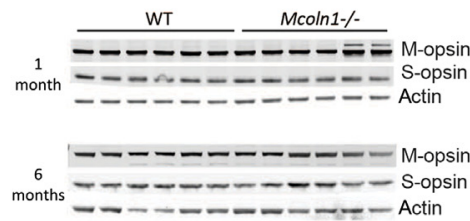

Figure 1 Thinning of the photoreceptor layer in Mcoln $1^{-/-}$mice. A: Representative images of hematoxylin and eosin-stained retinal sections from wild-type (WT) and Mcoln $1^{-/-}$mice at 1, 2, and 6 months of age. B: Quantitative analysis of hematoxylin and eosin-stained sections shows reduced thickness of the outer nuclear layer (ON) and outer photoreceptor segments (OS). Two-way analysis of variance analysis reveals significant effect of genotype on thickness of the outer nuclear layer $(F=173.7, P<0.001)$ and significant effect of genotype $(F=57.8, P<0.001)$ and age (F $=5.03, P=0.018)$ on thickness of outer segments. Asterisks represent Bonferroni posttest significance. C: Representative optical coherence tomography images of retina show reduced outer nuclear layer in Mcoln $1^{-/-}$retina at 1 month. Brackets indicate the outer nuclear layer (higher) and pigment epithelium (lower). D: Terminal deoxynucleotidyl transferase-mediated dUTP nick-end labeling (TUNEL) assay shows no difference in number of apoptotic cells between Mcoln1 ${ }^{-1}$ and WT littermate mice. Representative images of immunostaining (E) and immunoblotting (F) with antirhodopsin antibody shows reduced signal in Mcoln $1^{-/-}$mice. Representative images of peanut agglutinin (PNA) staining (G) and immunoblotting with cone-specific markers $\mathrm{S}$ - and M-opsins (H) show no difference between WT and Mcoln $1^{-1-}$ mice. ${ }^{*} P<0.05,{ }^{*} P P<0.01$, and $* * * P<0.001 . n=4$ (B, WT at 1 and 6 months and K0 at 6 months); $n=5$ (B, K0 at 1 and 2 months, and $\mathbf{D}, \mathrm{KO}$ at 2 months); $n=3$ (B, WT at 2 months, and $\mathbf{D}$, WT and $\mathrm{KO}$ at 1 month and WT at 2 months); $n=2$ (D, WT and K0 at 6 months). Scale bar $=50 \mu \mathrm{m}(\mathbf{A}, \mathbf{C}, \mathbf{E}$, and $\mathbf{G})$. IN, inner nuclear layer; IP, inner plexiform layer; IS/, inner segment of photoreceptors; $\mathrm{OP}$, outer plexiform layer; RNFL/GC, retinal nerve fiber layer/ganglion cell layer; RPE, retinal pigment epithelium.
(016-20001, 1:1000, rabbit polyclonal; Wako), anti-GFAP (number 3670, 1:2000, mouse monoclonal; Cell Signaling, Danvers, MA), anti- $\alpha$-tubulin (sc-8035, 1:10,000, mouse monoclonal; Santa Cruz Biotechnology), and anti- $\beta$-actin (A5441, 1:3000, mouse monoclonal; Sigma, La Jolla, CA). After incubation with primary antibody, the following secondary antibodies were applied: polyclonal goat anti-mouse or goat anti-rabbit IgG conjugated with IRDye 680 or IRDye 800 (both from LI-COR, Lincoln, NE). Protein bands were visualized using the Odyssey Infrared Imaging System and analyzed by Odyssey software version 3.0 (LICOR). In densitometric analysis, OD values were normalized by $\beta$-actin. All data were expressed as means \pm SEM. Data were analyzed statistically by $t$-test.

\section{EM Data}

Mice ( $n=3$ per genotype per time point) were euthanized by carbon dioxide inhalation; eyes were removed and fixed with half-strength Karnovsky's fixative for a minimum of 12 hours under refrigeration. After fixation, samples were rinsed with $0.1 \mathrm{~mol} / \mathrm{L}$ sodium cacodylate buffer, post-fixed with $2 \%$ osmium tetroxide in $0.1 \mathrm{~mol} / \mathrm{L}$ sodium cacodylate buffer, stained with $2 \%$ aqueous uranyl acetate, then dehydrated with graded ethyl alcohol solutions, transitioned with propylene oxide, and resin infiltrated in tEPON-812 epoxy resin (Tousimis, Rockville, MD) using an automated EMS Lynx 1 EM tissue processor (Electron Microscopy Sciences, Hatfield, PA). Processed tissues were oriented in tEPON812 epoxy resin and polymerized in silicone molds at $60^{\circ} \mathrm{C}$. Semithin cross sections for light microscopy were cut into sections $(1 \mu \mathrm{m}$ thick) and stained with $1 \%$ toluidine blue in $1 \%$ sodium tetraborate aqueous solution for assessment and screening regions of the tissue block face for dividing into thin sections. Ultrathin sections (60 to $90 \mathrm{~nm}$ thick) were cut from the epoxy block using a Leica EM UC7 ultramicrotome (Leica Microsystems) and a diamond knife, collected onto grids that were stained with aqueous $2 \%$ uranyl acetate and Sato's Lead citrate. Grids were imaged using a FEI Tecnai G2 Spirit transmission electron microscope (FEI, Hillsboro, OR), at $80 \mathrm{kV}$, interfaced with an AMT XR41 digital charge-coupled device camera (Advanced Microscopy Techniques, Danvers, MA) for digital tagged image file format file image acquisition. 
A

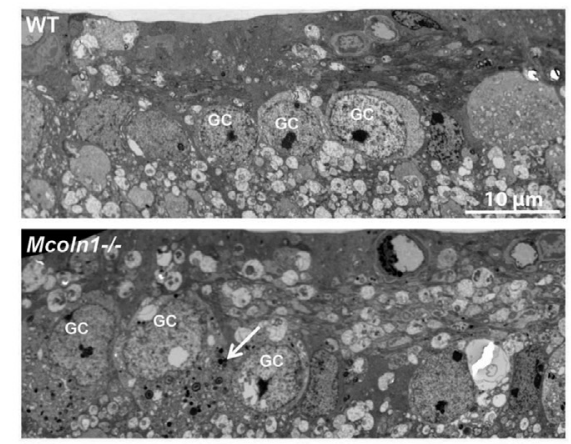

C

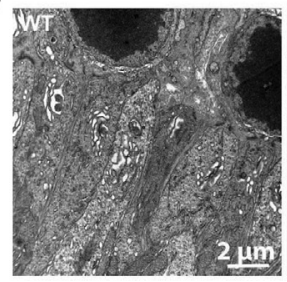

B
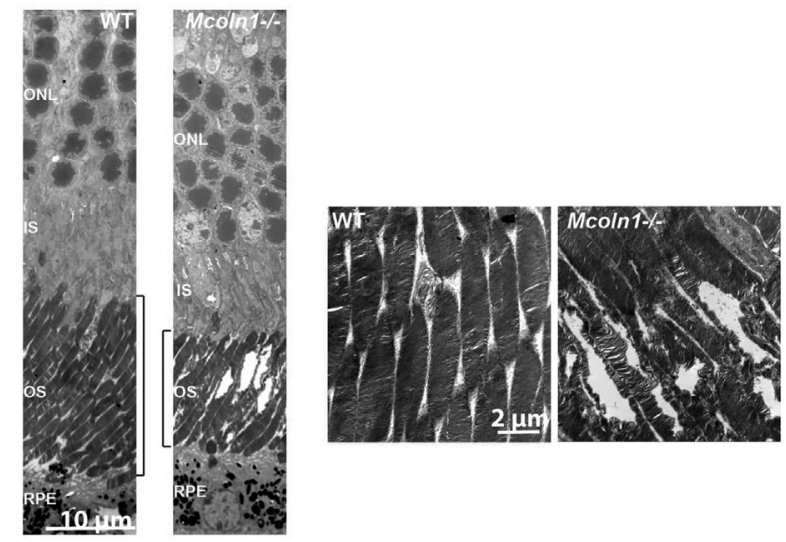

D

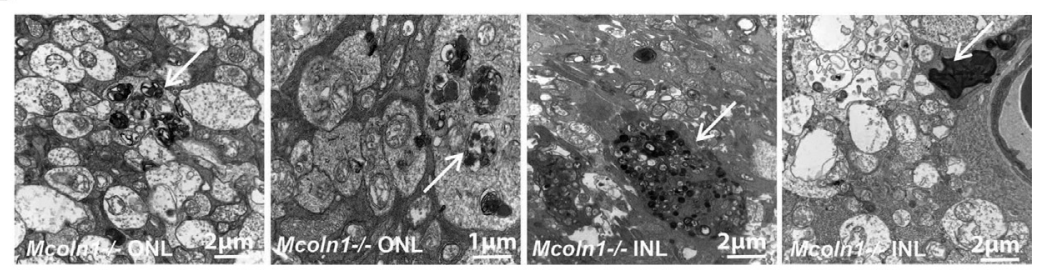

Figure 2 Electron microscopy findings in Mcoln1 ${ }^{-/-}$retina. A: Representative images of retinal ganglion layer. Arrow shows intracellular lysosomal storage inclusions in Mcoln1 $1^{-/}$retinal ganglion cells (GCS). B: Outer nuclear layer (ONL; left panels), note dysmorphic or ruptured opsin-containing disk membranes of photoreceptor outer segments (brackets), magnified in the right panels. C: Outer segments of photoreceptors (OS). Arrow shows example of aggregated lysosomes. D: Different types of storage inclusions in the retinal plexiform layers of Mcoln1 ${ }^{-/}$mice as indicated by arrows. INL, inner nuclear layer; IS, inner segment of photoreceptors; RPE, retinal pigment epithelium; WT, wild type.

Digital image analysis was performed using Fiji software. For myelin sheath thickness analysis, g ratio (axon diameter/fiber diameter, where fiber diameter is a sum of axon diameter and myelin sheath thickness) has been calculated as follows: for each myelinated axon in the field of view (magnification $\times 2900$ ), two selections, one is around the internal surface of the sheath (corresponding to axon) and second is around the outer edge of the sheath (corresponding to fiber), were drawn and the corresponding area was measured. The diameters of each axon and corresponding fiber and corresponding g ratio were calculated in Excel 2010 (Microsoft Corp., Redmond, WA). The normality of all data sets was tested using the Kolmogorov-Smirnov method. Nonparametric data are presented as median values with interquartile ranges. An extended Wilcoxon rank-sum test that accommodates clustered data ${ }^{38}$ was used for comparisons between control and $M \operatorname{coln} 1^{-1-}$ groups for nonnormally distributed data. The statistical significance threshold was set at $P<0.05$.

\section{Results}

Examination of hematoxylin and eosin-stained retinal sections of $M c o l n 1^{-1-}$ and WT littermates revealed significant thinning of the photoreceptor layer by $46 \%$ in Mcoln $1^{-/-}$retina at 1 month of age, which did not progress during the course of disease (two-way analysis of variance:
$\mathrm{F}_{\text {genotype }}=173.67 ; P_{\text {genotype }}<0.0001 ; \mathrm{F}_{\text {age }}=0.08 ;$ $P_{\text {age }}=0.92$; Bonferroni post tests: $P<0.001$ for all time points) (Figure 1, A-C). Together with the thinning of the outer nuclear layer that is composed of perikaryons of rods and cones, we observed a significant reduction by $39 \%$ in the length of photoreceptor outer segments in Mcoln1 $1^{-1-}$ mice (Figure 1B), which partially improved with age (two-way analysis of variance: $\mathrm{F}_{\text {genotype }}=57.78$; $P_{\text {genotype }}<0.0001 ; \mathrm{F}_{\text {age }}=5.3 ; P_{\text {age }}=0.0176 ;$ Bonferroni post tests: $P<0.001$ at 1 and 2 months; $P<0.05$ at 3 months of age) (Figure 1B). To determine whether loss of mucolipin-1 causes progressive retinal degeneration in the course of disease, we performed TUNEL assay on 1-, 2-, and 6-month-old retinas. TUNEL assay showed no difference in the number of apoptotic cells between genotypes at any of the examined time points (Figure 1D). These data rule out ongoing cell death in $M \operatorname{coln} 1^{-/-}$retina during the course of disease and imply that loss of mucolipin- 1 affects retinal development. To determine whether rods and cones are equally affected, we performed immunoanalysis with cell-specific markers: rhodopsin for rods and peanut agglutinin and cone opsins for cones. Immunostaining with rhodopsin revealed thinning in the rod outer segments (ROS) in Mcoln1 ${ }^{-1-}$ mice that was most pronounced at 1 month of age (Figure 1E) and corresponds to the measurement of outer segment thickness on hematoxylin and eosin-stained sections (Figure 1B). Similarly, we observed a significant reduction of rhodopsin levels by $50 \%$ at 1 and 


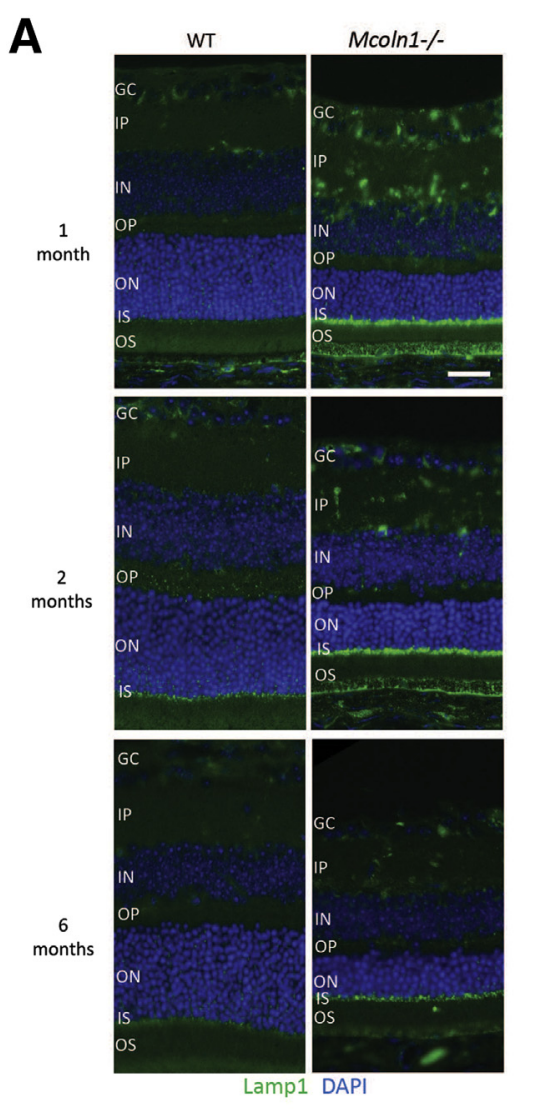

E
B
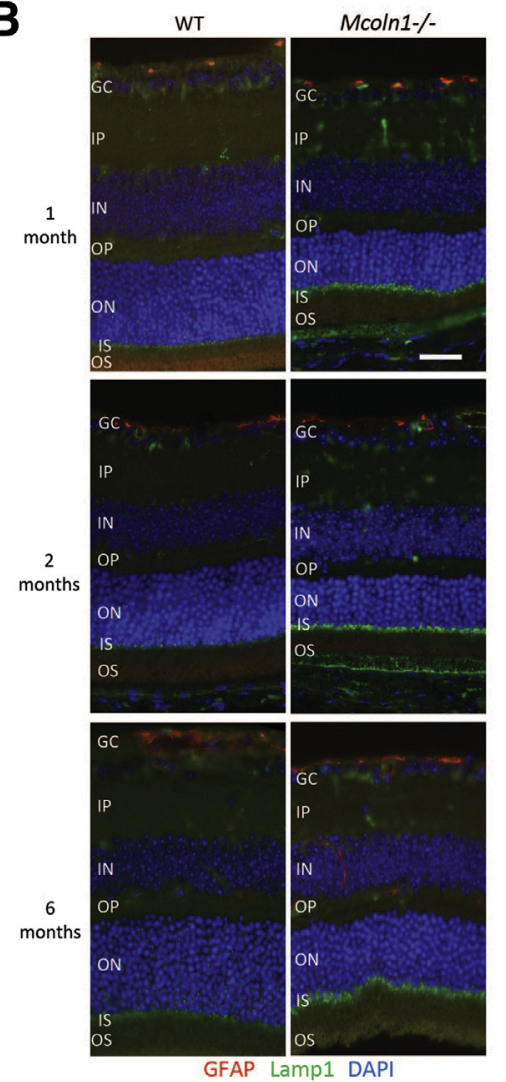

Mcoln1-/-

Calbindin Lamp1 DAPI
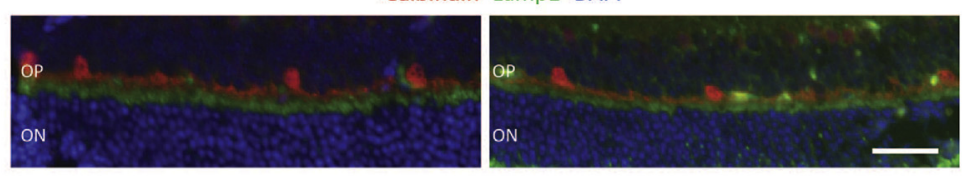

PKC $\alpha$ Lamp1 DAPI
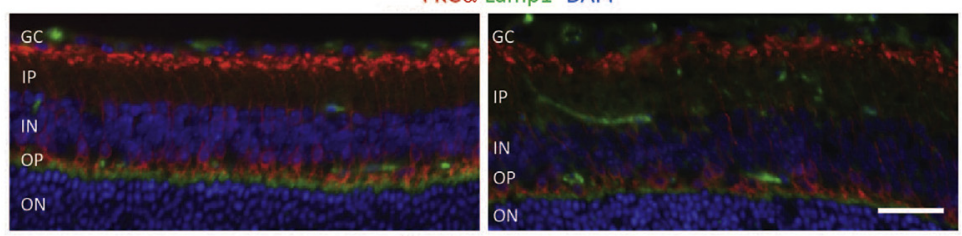

Parvalbumin Lamp1 DAPI

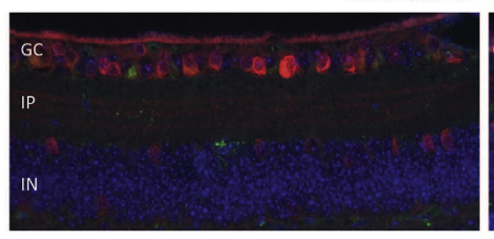

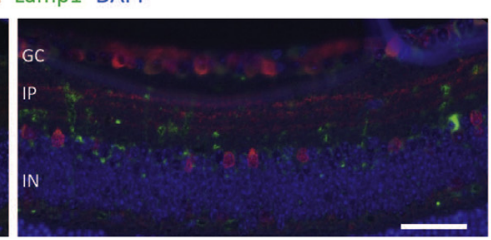

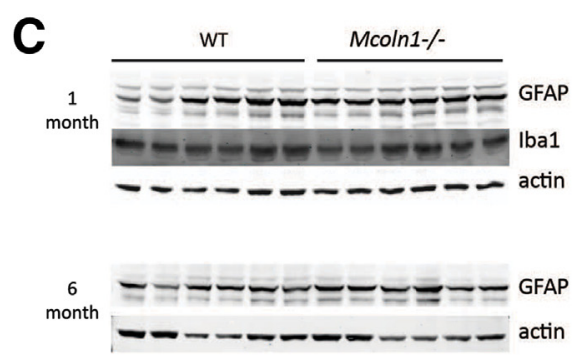

D

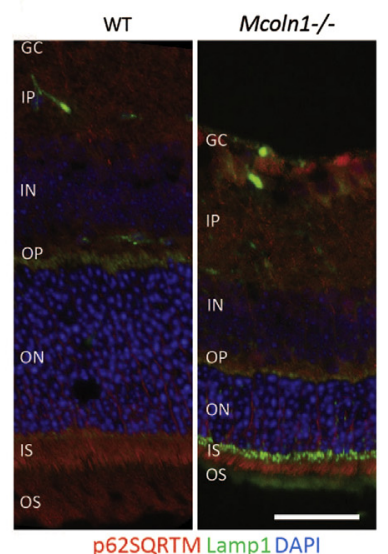

Figure 3 Lysosomal alterations in Mcoln $1^{-/-}$ retina. A: Representative images of Lamp1 immunohistochemistry show increase in staining in $M c o l n 1^{-/-}$retina, affecting retinal ganglion cells, inner plexiform layer, and inner segments of photoreceptors. B: Immunostaining with astrocyte and Muller glia marker, glial fibrillary acidic protein (GFAP), shows no changes in Mcoln1 ${ }^{-/-}$retina compared with wild type (WT). C: Western blot analyses on whole eye homogenates show no changes in levels of GFAP and Ibal in Mcoln $1^{-/-}$versus WT eyes. D: Costaining of Lamp-1 and p62/sequestosome 1 protein aggregates shows no colocalization in Mcoln $1^{-/-}$retina. E: Coimmunostaining of Lamp1 with calbindin, protein kinase $C-\alpha$, and parvalbumin shows no pronounced colocalization of these cell type markers with Lamp1. Scale bars: $20 \mu \mathrm{m}$ (A, B, and $\mathbf{E}) ; 30 \mu \mathrm{m}(\mathbf{D}) . \mathrm{GC}$, ganglion cell layer; IN, inner nuclear layer; IP, inner plexiform layer; IS, inner segments of photoreceptors; $\mathrm{ON}$, outer nuclear layer; $\mathrm{OP}$, outer plexiform layer; $\mathrm{OS}$, outer segments of photoreceptors.
$70 \%$ at 6 months of age on Western blot analysis (Figure 1F). No changes were observed with the cone cell markers by either immunohistochemistry or immunoblotting (Figure 1, G and $\mathrm{H}$ ), implying that rods, not cones, are mainly affected by the loss of mucolipin-1.

The most prominent pathological hallmark of MLIV is the formation of lysosomal storage inclusions called compound bodies that are filled with lipids and polysaccharides. ${ }^{39}$
Ultrastructural analysis of 1-month-old retinas using transmission EM revealed the appearance of storage inclusions in lysosomes of $M c o l n 1^{-/-}$mice. These storage bodies were present in retinal ganglion cells (Figure 2A), inner segments of photoreceptor cells (Figure 2C), and cell processes forming both the inner and outer photoreceptor layers (Figure 2D). Storage inclusions that we observed in the $M \operatorname{coln} 1^{-/-}$retina had typical membranous electron-dense content similar to 
A

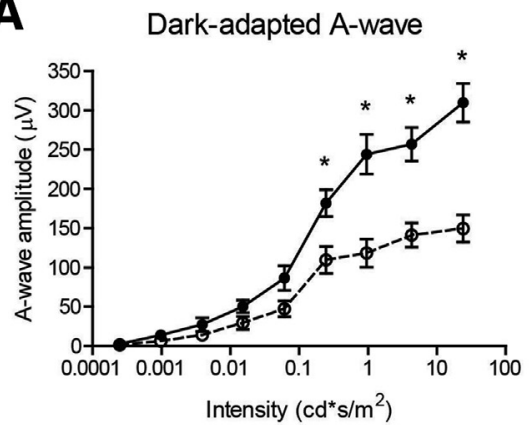

C

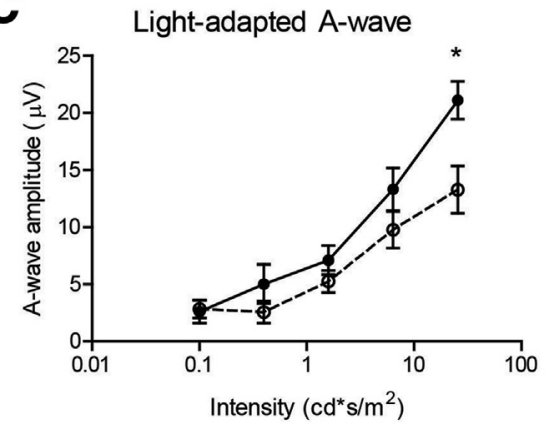

B

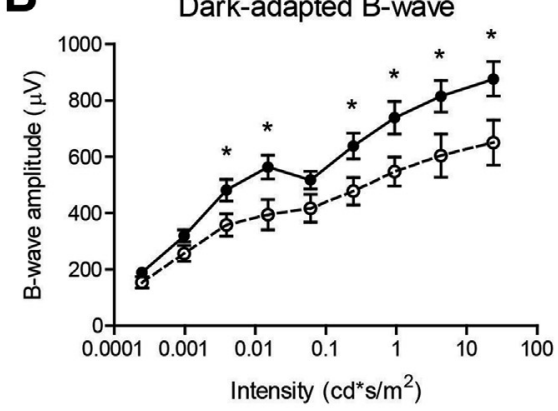

D

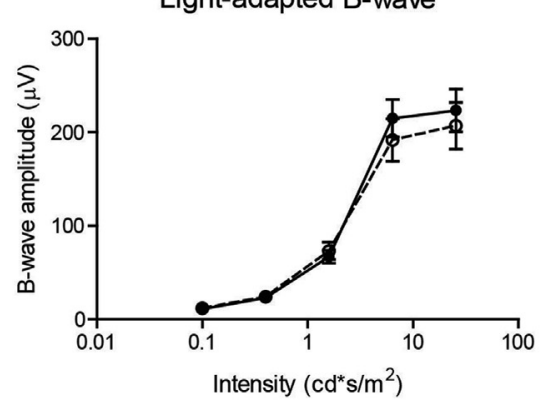

Figure 4 Effect of mucolipin-1 loss on visual function in mice. Amplitudes of the a-wave ( $\mathbf{A}$ and $\mathbf{C}$ ) and $\mathbf{b}$-wave (B and $\mathbf{D}$ ) of the dark-adapted (A and $\mathbf{B})$ and light-adapted (C and $\mathbf{D})$ electroretinography (ERG) in Mcoln $1^{-1-}$ (KO; open circles) and wild-type (WT; closed circles) littermates at 1 month of age plotted as function of log-flash intensity for each group. Scotopic ERGs were recorded at nine different flash intensities; photopic ERGs were recorded at five stimulus intensities. The means \pm SEM $a$ - and $b$-wave amplitudes are plotted (A-D). $n=14$ (A-D, WT); $n=13$ (A-D, K0). ${ }^{*} P<0.05$ (t-test). inclusions reported in both $M \operatorname{coln} 1^{-1-}$ mouse brain and patient cells. ${ }^{30,40,41}$ EM confirmed the thinning of the outer nuclear layer and outer photoreceptor segments in $M c o l n 1^{-1-}$ mice, and revealed dysmorphic opsin-containing disk membranes in the ROS (Figure 2B).

Immunostaining for a lysosomal marker Lamp1 revealed enlargement and aggregation of lysosomes in the $M \operatorname{coln} 1^{-1-}$ retina (Figure 3A). Similar to our ultrastructural findings, Lamp1-positive aggregates were prominent in photoreceptors, retinal ganglion cells, and inner and outer plexiform layers. In photoreceptor cells, Lamp1-positive organelles aggregated in the inner segments. Immunostaining with markers for amacrine, horizontal, or bipolar cells (parvalbumin, calbindin, and protein kinase $\mathrm{C}-\alpha$, correspondingly) revealed no changes in morphology between WT and Mcoln $1^{-1-}$ mice, and no overt colocalization of these markers with Lamp1-positive lysosomal aggregates (Figure 3E and Supplemental Figure S1).

One of the most striking phenotypes observed in the Mcoln $1^{-/-}$mouse brain is early activation of microglia and astrocytes. $^{28}$ To determine whether glial cells are involved in the observed retinal pathology, we performed immunoanalysis with markers for astrocytes and Muller glia (GFAP) (Figure 3, B and C, and Supplemental Figure S2) and microglia/monocytes (Iba1) (Figure 3C and Supplemental Figure S3). Interestingly, unlike in the brain, no differences in glial cell morphology, staining intensity, or distribution were observed for either GFAP or Ibal between genotypes. Accordingly, we observed no significant difference in the levels of GFAP and Iba1 by Western blot analysis (Figure 3C). Protein aggregates labeled by anti-p62/ sequestosome 1 have been previously reported in the Mcoln $1^{-1-}$ brain. ${ }^{29}$ However, in the retina, coimmunostaining of p62/sequestosome 1 with Lamp1 failed to reveal accumulation of protein aggregates in the lysosomes (Figure 3D), suggesting that composition of accumulating storage material in MLIV depends on metabolic specifics and, thus, may differ in various types of cells and tissues.

To determine whether the observed structural changes in the retina caused by lack of mucolipin-1 alter visual acuity, we performed ERG in Mcoln $1^{-1-}$ and WT littermate mice at 1 month of age. We tested the ERG in both scotopic (darkadapted) and photopic (light-adapted) conditions (Figure 4). We observed significantly lower a-wave amplitudes at higher stimulus intensities in both dark- and light-adapted animals, consistent with the loss of photoreceptors. We also observed significantly lower scotopic b-wave amplitudes in $M \operatorname{coln} 1^{-1-}$ retina. Under photopic conditions, the a-wave differences were statistically significant only at the highest intensity. At that same intensity, the differences in b-wave amplitude between genotypes did not attain statistical significance. This may be because of the fact that the b-wave is nearing saturation at this intensity, but the a-wave continues to increase. Cone-bipolar cell responses that drive the photopic b-wave can saturate at lower intensities than cone responses. $^{42}$

Dysmyelination in the brain is one of the major phenotypic characteristics in both MLIV patients and Mcoln $1^{-1-}$ mice, ${ }^{28,43}$ and optic nerve pallor has been reported in MLIV patients. ${ }^{4}$ Therefore, we examined ultrastructure of the optic nerve in $M \operatorname{coln} 1^{-1-}$ mice at 1 and 6 months of age (Figure 5). At 1 month, we observed no difference in myelination, expressed as g ratio (axon diameter/fiber diameter, where fiber diameter is the sum of axon diameter and myelin sheath thickness $)(P=0.62$, Wilcoxon rank-sum test $)$, density $(P=$ $0.36, t$-test $)$, and percentage of myelinated axons $(P=0.38$, 
A
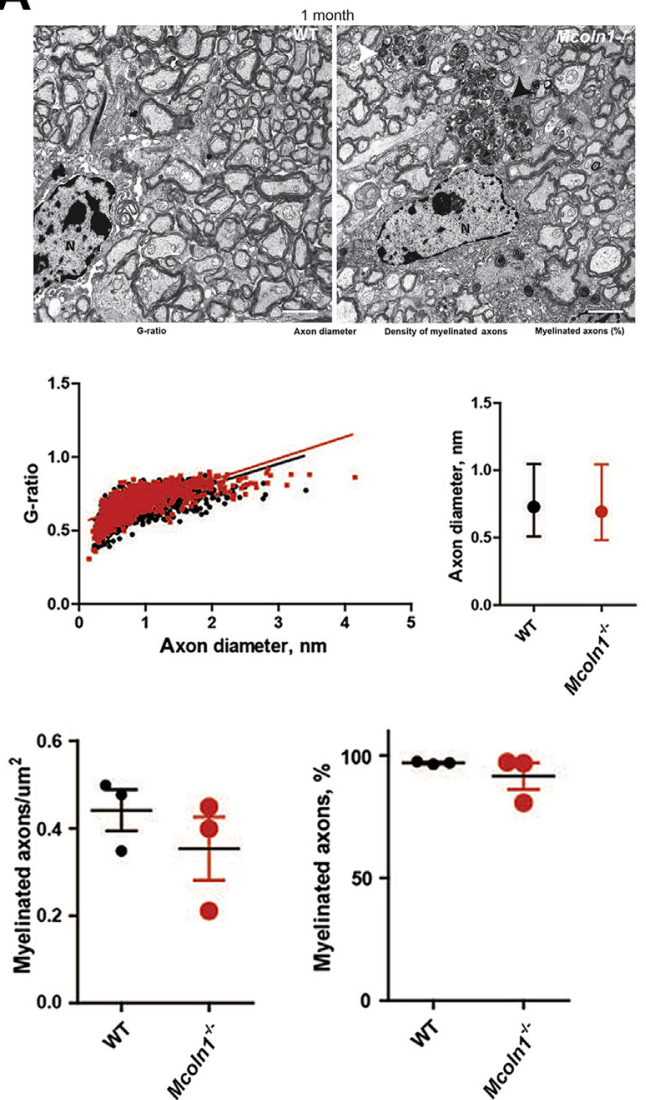

B
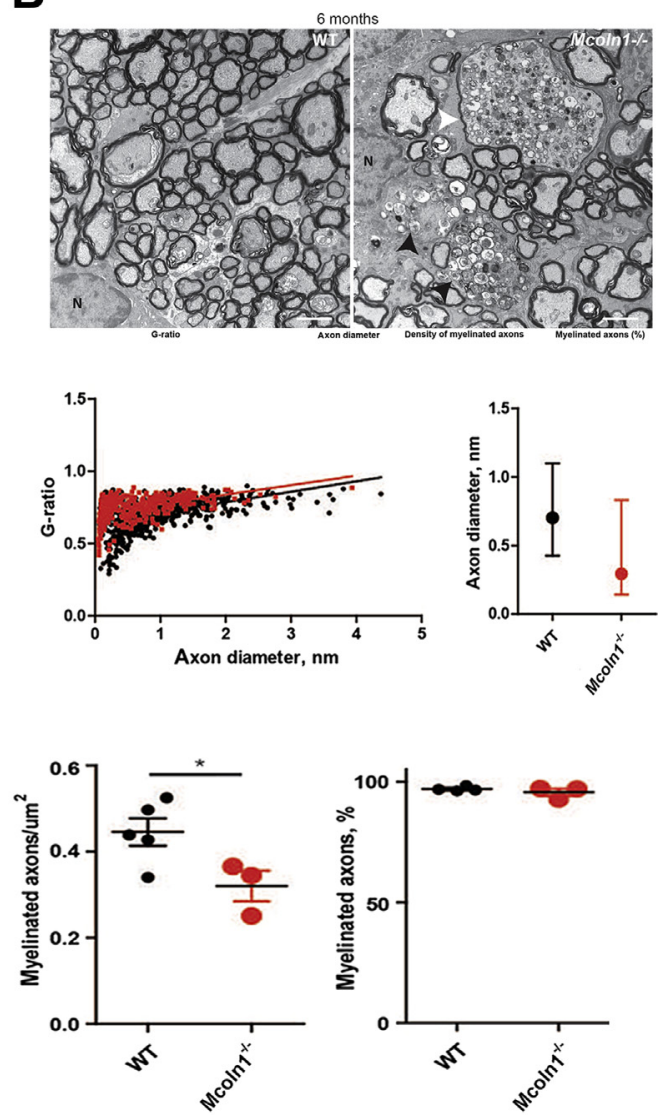

$t$-test) (Figure 5A). However, we observed large aggregates of storage inclusions in the $M \operatorname{coln} 1^{-1-}$ optic nerve that were present within axons and in the soma (of glial cells) (Figure 5A). At 6 months, we found decreased density of myelinated axons in the optic nerve of $M \operatorname{coln} 1^{-1-}$ mice $(P=0.045, t$-test $)$, which indicates lateonset axonal degeneration (Figure 5B). However, no significant difference in $\mathrm{g}$ ratio $(P=0.15$, Wilcoxon ranksum test), axon diameter $(P=0.47$, Wilcoxon rank-sum test), or percentage of myelinated axons $(P=0.37, t$-test $)$ was observed.

\section{Discussion}

Visual impairment is a characteristic manifestation of MLIV in humans. Visual perception declines during the course of the disease because of corneal clouding, retinal degeneration, and optic nerve deterioration, which progresses to blindness in the second decade of life. ${ }^{4,5}$ To better understand retinal pathology because of the loss of mucolipin-1, we characterized the retinal phenotype in the $M \operatorname{coln} 1^{-1-}$ mouse model, shown to be an accurate phenotypic model of the disease, ${ }^{27-30}$ at the ages of 1,2 , and 6 months. These time points are referred to as presymptomatic, early symptomatic, and late stage, respectively, on the basis of an evaluation of neurological function. Our previous studies show onset of early motor and cognitive impairment in the Mcoln $1^{-1-}$ mouse as early as 2 months. ${ }^{28}$ In the current study, we report thinning of the photoreceptor cell layer in Mcoln $1^{-1-}$ retina that was evident at 1 month of age (ie, before the onset of observable neurological deficits). Our histological and Western blot data suggest that among photoreceptors, rods are mainly affected by the loss of

Figure 5 0ptic nerve ultrastructure in Mcoln1 ${ }^{-/-}$mice. A: Representative electron micrographs of the optic nerve in 1-month-old control [wild type (WT)] and Mcoln1 $1^{-/-}$[knockout (KO)] mice showing accumulation of the storage inclusions in axons and cytoplasm of glial cells (black arrowhead). The $\mathrm{g}$ ratio (axon diameter/fiber diameter) plotted against axon diameter shows no difference in myelination in Mcoln1 ${ }^{-1-}$ (1293 axons; three mice) compared with wild-type (1287 axons; three mice), $P=0.62$ (clustered rank-sum test). Comparison of axon diameters reveals no significant difference between WT and KO littermates. Quantification of myelinated axon density and percentage of myelinated axons reveals no difference between WT and KO littermates, $P_{\text {density }}=0.36$ ( $t$-test); $P_{\% \text { of myelinated axons }}=0.38$ ( $t$-test). B: Representative electron micrographs of the optic nerve in 6-month-old WT and KO mice show accumulation of the storage inclusions in axons (white arrowhead) and cytoplasm of glial cells (black arrowhead). The $\mathrm{g}$ ratio (axon diameter/fiber diameter) plotted against axon diameter shows no difference in myelination in Mcoln1-1(466 axons; three mice) compared with wild type (1037 axons; five mice), $P=0.15$ (clustered rank-sum test). Comparison of axon diameters reveals no significant difference between WT and KO littermates. Quantification of myelinated axon density reveals significantly reduced density in $\mathrm{KO}$ mice, indicating axonal pathology, whereas percentage of myelinated axons between WT and KO littermates remains unchanged, $P=0.37$ ( $t$-test). Data presented as median values with interquartile range, by clustered rank-sum test $[P=0.12(\mathbf{A})$ and $P=0.47(\mathbf{B})] .{ }^{\star} P<0.05$. N, nuclei of glial cells (astrocytes). 
mucolipin-1, as shown by reduced levels of rhodopsin and dysmorphic ultrastructure of the outer segments. Our functional ERG data support histological observations. We observed reduced response to light stimulation in the darkadapted (scotopic) recordings, as indicated by reduced amplitudes of both a- and b-waves in $M \operatorname{coln} 1^{-1-}$ mice at 1 month of age compared with their WT littermates. Interestingly, thinning of the photoreceptor layer did not progress during the course of the disease. The a-wave reflects photoreceptor responses and is consistent with more significant loss of rods than cones. In Mcoln $1^{-1-}$ mice, scotopic a-wave amplitude was diminished over a wider range of intensities than photopic a-wave, which was reduced only at the highest test intensity. The b-wave reflects outer nuclear bipolar cell responses, and diminished b-wave amplitudes in $M \operatorname{coln} 1^{-1-}$ mice are likely to be a secondary consequence of the loss of photoreceptor function, as reflected by the diminished a-wave. Interestingly, we observed improvement in the thickness of the disk membrane-containing outer segments in $M \operatorname{coln} 1^{-1-}$ retina with age. These data suggest that retinal dystrophy in the Mcoln $1^{-/-}$mouse is a result of an early developmental insult affecting mainly rods, with no progressive loss of photoreceptors.

Immunohistochemistry and EM analysis revealed shortened and disrupted ROS in the Mcoln $1^{-1-}$ retina, which suggest that mucolipin-1 plays a role in the regulation of disk membrane biogenesis and ROS assembly or renewal. ROS are composed of the stacks of membranous disks bearing the light transduction machinery. In rodents, outer segments begin to form 10 days after birth and attain their mature length between 4 and 6 weeks of age. ${ }^{44}$ New disks are assembled in the ciliary plasmalemma at the base of the outer segments. In the adult retina, constant renewal of disks maintains the length of ROS because of balancing of the shedding disks at the distal tip and assembly of new disks at the base of the outer segment. According to a freezesubstitution EM study, membranous disks are formed by the fusion and compression of small pinocytotic vesicles at the base of the outer segment. ${ }^{45}$ Thus, given the widely accepted role of mucolipin-1 in various membranetrafficking and fusion events, ${ }^{18,19,23,46,47}$ the observed inefficient developmental assembly of ROS in the $M \operatorname{coln} 1^{-1-}$ mouse (Figure 1) could be explained by aberrant membrane fusion because of the loss of mucolipin-1.

In correlation with abundant lysosomal storage inclusions detected by EM, we observed increased Lamp1 signal in the $M c o l n 1^{-1-}$ retina, indicating enlargement of lysosomes because of accumulation of storage material. In photoreceptors, lysosomes were aggregated in the inner segments in $M \operatorname{coln} 1^{-1-}$ mice, the region of the cell where lysosome-dependent autophagic degradation of the outer segments normally takes place. ${ }^{48}$ Autophagic-lysosomal degradation is important for maintaining ROS and adaptation to light. ${ }^{48}$ Most interestingly, rods and cones display a different response to the autophagy activator rapamycin, indicating that the autophagy process may play distinct roles in these cells. ${ }^{49}$ Although in rods, activation of autophagy in albino mouse light injury model increased survival and function, in cones, it caused excessive vacuole formation and functional decline. ${ }^{49}$ Given the role of autophagy in the maintenance of the outer segments and the differential regulation of this process in rods and cones, inhibition of the autophagy-lysosomal pathway because of loss of mucolipin-1 can explain the pattern of photoreceptor injury in $M \operatorname{coln} 1^{-1-}$ mice that we describe herein. Supporting this hypothesis, defects in autophagy because of loss of mucolipin-1 have been reported previously. $23,31,50$

EM analysis of the Mcoln $1^{-1-}$ mouse retina revealed numerous storage bodies in all retinal layers and in the optic nerve that resemble typical storage bodies reported in both human and mouse brain. ${ }^{30,40,41}$ In the optic nerve axons of $M \operatorname{coln} 1^{-1-}$ mice, we observed formation of large aggregates of electron dense inclusions, vacuoles, and mitochondria at 1 and 6 months of age. These aggregates of storage inclusions were similar to the previously reported axonal spheroids in cerebellar Purkinje cells,${ }^{29}$ indicating axonal dystrophy in the optic nerve of $M \operatorname{coln} 1^{-1-}$ mice. Accumulation of axonal spheroids leads to axonal degeneration later in disease, as shown by decreased axon density in the optic nerve of $M \operatorname{coln} 1^{-1-}$ mice at 6 months of age.

The eye pathology in $M \operatorname{coln} 1^{-1-}$ mice resembles the ophthalmic features described in human MLIV patients. Both humans and mice show loss of photoreceptors in the retina, progressive optic nerve degeneration, and intracellular storage inclusions. Interestingly, as reported earlier, $M \operatorname{coln} 1^{-1-}$ mice display no corneal pathology despite this being a hallmark of the human disease. ${ }^{30}$ Although the etiology of this phenomenon is not clear, this discrepant phenotype has also been observed in other mouse models of lysosomal storage diseases, such as mucopolysaccharidosis I, mucopolysaccharidosis IV, and GM1 gangliosidosis, ${ }^{51-53}$ and could reflect different proteoglycan biochemistry in the mouse cornea. Another interesting observation is the difference in disease progression between human and mice. Although there are only a few longitudinal studies of eye pathology in MLIV, they demonstrate progressive worsening of ophthalmic features in patients. ${ }^{2,4,51}$ Data in $M c o l n 1^{-1-}$ mice revealed that thinning of photoreceptor layer and ERG responses were not progressive in the course of disease. Nevertheless, despite the altered time course, recapitulation of the photoreceptor and optic nerve pathological features of MLIV in $M \operatorname{coln} 1^{-1-}$ mice makes it a good model for preclinical gene therapy testing for this devastating disease.

Overall, these findings provide the first comprehensive characterization of the eye phenotype in the mouse model of mucolipidosis IV, revealing early thinning of the photoreceptor layer, disturbed lysosomal morphology, massive widespread intracellular storage deposition, and reduced ERG responses as the major pathological features, providing a set of outcome measures for preclinical trials and setting a stage for further mechanistic investigations of the mucolipin-1 role in retinal function. 


\section{Acknowledgments}

We thank Philip Seifert (Schepen's Eye Research Institute electron microscopy core) for electron microscopy sample preparation and image acquisition, Oscar Morales for conducting electroretinographic recording procedures, and Dr. Igor Nasonkin for critical reading of the manuscript and useful discussion.

\section{Supplemental Data}

Supplemental material for this article can be found at http://dx.doi.org/10.1016/j.ajpath.2015.09.017.

\section{References}

1. Berman ER, Livni N, Shapira E, Merin S, Levij IS: Congenital corneal clouding with abnormal systemic storage bodies: a new variant of mucolipidosis. J Pediatr 1974, 84:519-526

2. Abraham FA, Brand N, Blumenthal M, Merin S: Retinal function in mucolipidosis IV. Ophthalmologica 1985, 191:210-214

3. Riedel KG, Zwaan J, Kenyon KR, Kolodny EH, Hanninen L, Albert DM: Ocular abnormalities in mucolipidosis IV. Am J Ophthalmol 1985, 99:125-136

4. Smith JA, Chan CC, Goldin E, Schiffmann R: Noninvasive diagnosis and ophthalmic features of mucolipidosis type IV. Ophthalmology 2002, 109:588-594

5. Wakabayashi K, Gustafson AM, Sidransky E, Goldin E: Mucolipidosis type IV: an update. Mol Genet Metab 2011, 104:206-213

6. Bargal R, Avidan N, Ben-Asher E, Olender Z, Zeigler M, Frumkin A, Raas-Rothschild A, Glusman G, Lancet D, Bach G: Identification of the gene causing mucolipidosis type IV. Nat Genet 2000, 26: $118-123$

7. Bassi MT, Manzoni M, Monti E, Pizzo MT, Ballabio A, Borsani G: Cloning of the gene encoding a novel integral membrane protein, mucolipidin, and identification of the two major founder mutations causing mucolipidosis type IV. Am J Hum Genet 2000, 67: $1110-1120$

8. Slaugenhaupt SA, Acierno JS Jr, Helbling LA, Bove C, Goldin E, Bach G, Schiffmann R, Gusella JF: Mapping of the mucolipidosis type IV gene to chromosome 19p and definition of founder haplotypes. Am J Hum Genet 1999, 65:773-778

9. Sun M, Goldin E, Stahl S, Falardeau JL, Kennedy JC, Acierno JS Jr, Bove C, Kaneski CR, Nagle J, Bromley MC, Colman M, Schiffmann R, Slaugenhaupt SA: Mucolipidosis type IV is caused by mutations in a gene encoding a novel transient receptor potential channel. Hum Mol Genet 2000, 9:2471-2478

10. Slaugenhaupt SA: The molecular basis of mucolipidosis type IV. Curr Mol Med 2002, 2:445-450

11. Dong XP, Cheng X, Mills E, Delling M, Wang F, Kurz T, Xu H: The type IV mucolipidosis-associated protein TRPML1 is an endolysosomal iron release channel. Nature 2008, 455:992-996

12. Dong XP, Wang X, Shen D, Chen S, Liu M, Wang Y, Mills E, Cheng X, Delling M, Xu H: Activating mutations of the TRPML1 channel revealed by proline-scanning mutagenesis. J Biol Chem 2009, 284:32040-32052

13. Xu H, Delling M, Li L, Dong X, Clapham DE: Activating mutation in a mucolipin transient receptor potential channel leads to melanocyte loss in varitint-waddler mice. Proc Natl Acad Sci U S A 2007, 104: $18321-18326$

14. LaPlante JM, Falardeau JL, Brown EM, Slaugenhaupt SA, Vassilev PM: The cation channel mucolipin-1 is a bifunctional protein that facilitates membrane remodeling via its serine lipase domain. Exp Cell Res 2011, 317:691-705

15. Kiselyov K, Chen J, Rbaibi Y, Oberdick D, Tjon-Kon-Sang S, Shcheynikov N, Muallem S, Soyombo A: TRP-ML1 is a lysosomal monovalent cation channel that undergoes proteolytic cleavage. J Biol Chem 2005, 280:43218-43223

16. LaPlante JM, Ye CP, Quinn SJ, Goldin E, Brown EM, Slaugenhaupt SA, Vassilev PM: Functional links between mucolipin1 and $\mathrm{Ca} 2+$-dependent membrane trafficking in mucolipidosis IV. Biochem Biophys Res Commun 2004, 322:1384-1391

17. Manzoni M, Monti E, Bresciani R, Bozzato A, Barlati S, Bassi MT, Borsani G: Overexpression of wild-type and mutant mucolipin proteins in mammalian cells: effects on the late endocytic compartment organization. FEBS Lett 2004, 567:219-224

18. Treusch S, Knuth S, Slaugenhaupt SA, Goldin E, Grant BD, Fares H: Caenorhabditis elegans functional orthologue of human protein h-mucolipin-1 is required for lysosome biogenesis. Proc Natl Acad Sci U S A 2004, 101:4483-4488

19. Chen CS, Bach G, Pagano RE: Abnormal transport along the lysosomal pathway in mucolipidosis, type IV disease. Proc Natl Acad Sci U S A 1998, 95:6373-6378

20. Pryor PR, Reimann F, Gribble FM, Luzio JP: Mucolipin-1 is a lysosomal membrane protein required for intracellular lactosylceramide traffic. Traffic 2006, 7:1388-1398

21. Piper RC, Luzio JP: CUPpling calcium to lysosomal biogenesis. Trends Cell Biol 2004, 14:471-473

22. Venkatachalam K, Long AA, Elsaesser R, Nikolaeva D, Broadie K, Montell C: Motor deficit in a Drosophila model of mucolipidosis type IV due to defective clearance of apoptotic cells. Cell 2008, 135: $838-851$

23. Vergarajauregui S, Connelly PS, Daniels MP, Puertollano R: Autophagic dysfunction in mucolipidosis type IV patients. Hum Mol Genet 2008, 17:2723-2737

24. LaPlante JM, Sun M, Falardeau J, Dai D, Brown EM, Slaugenhaupt SA, Vassilev PM: Lysosomal exocytosis is impaired in mucolipidosis type IV. Mol Genet Metab 2006, 89:339-348

25. Lima WC, Leuba F, Soldati T, Cosson P: Mucolipin controls lysosome exocytosis in Dictyostelium. J Cell Sci 2012, 125 $2315-2322$

26. Gilliam JC, Wensel TG: TRP channel gene expression in the mouse retina. Vision Res 2011, 51:2440-2452

27. Chandra M, Zhou H, Li Q, Muallem S, Hofmann SL, Soyombo AA: A role for the Ca2+ channel TRPML1 in gastric acid secretion, based on analysis of knockout mice. Gastroenterology 2011, 140:857-867

28. Grishchuk Y, Sri S, Rudinskiy N, Ma W, Stember KG, Cottle MW, Sapp E, Difiglia M, Muzikansky A, Betensky RA, Wong AM, Bacskai BJ, Hyman BT, Kelleher RJ, Cooper JD, Slaugenhaupt SA: Behavioral deficits, early gliosis, dysmyelination and synaptic dysfunction in a mouse model of mucolipidosis IV. Acta Neuropathol Commun 2014, 2:133

29. Micsenyi MC, Dobrenis K, Stephney G, Pickel J, Vanier MT, Slaugenhaupt SA, Walkley SU: Neuropathology of the Mcoln1(-/-) knockout mouse model of mucolipidosis type IV. J Neuropathol Exp Neurol 2009, 68:125-135

30. Venugopal B, Browning MF, Curcio-Morelli C, Varro A, Michaud N, Nanthakumar N, Walkley SU, Pickel J, Slaugenhaupt SA: Neurologic, gastric, and opthalmologic pathologies in a murine model of mucolipidosis type IV. Am J Hum Genet 2007, $81: 1070-1083$

31. Curcio-Morelli C, Charles FA, Micsenyi MC, Cao Y, Venugopal B, Browning MF, Dobrenis K, Cotman SL, Walkley SU, Slaugenhaupt SA: Macroautophagy is defective in mucolipin-1deficient mouse neurons. Neurobiol Dis 2010, 40:370-377

32. Mattapallil MJ, Wawrousek EF, Chan CC, Zhao H, Roychoudhury J, Ferguson TA, Caspi RR: The Rd8 mutation of the Crb1 gene is present in vendor lines of C57BL/6N mice and embryonic stem cells, 
and confounds ocular induced mutant phenotypes. Invest Ophthalmol Vis Sci 2012, 53:2921-2927

33. Jelcick AS, Yuan Y, Leehy BD, Cox LC, Silveira AC, Qiu F, Schenk S, Sachs AJ, Morrison MA, Nystuen AM, DeAngelis MM, Haider NB: Genetic variations strongly influence phenotypic outcome in the mouse retina. PLoS One 2011, 6:e21858

34. Haider NB, Demarco P, Nystuen AM, Huang X, Smith RS, McCall MA, Naggert JK, Nishina PM: The transcription factor Nr2e3 functions in retinal progenitors to suppress cone cell generation. Vis Neurosci 2006, 23:917-929

35. Goldman SA, Nedergaard M, Windrem MS: Glial progenitor cellbased treatment and modeling of neurological disease. Science 2012, 338:491-495

36. Cruz NM, Yuan Y, Leehy BD, Baid R, Kompella U, DeAngelis MM, Escher P, Haider NB: Modifier genes as therapeutics: the nuclear hormone receptor Rev Erb alpha (Nr1d1) rescues Nr2e3 associated retinal disease. PLoS One 2014, 9:e87942

37. Mollema NJ, Yuan Y, Jelcick AS, Sachs AJ, von Alpen D, Schorderet D, Escher P, Haider NB: Nuclear receptor Rev-erb alpha (Nr1d1) functions in concert with Nr2e3 to regulate transcriptional networks in the retina. PLoS One 2011, 6:e17494

38. Datta S, Satten GA: Rank-sum tests for clustered data. J Am Stat Assoc 2005, 100:908-915

39. Chitayat D, Meunier CM, Hodgkinson KA, Silver K, Flanders M, Anderson IJ, Little JM, Whiteman DA, Carpenter S: Mucolipidosis type IV: clinical manifestations and natural history. Am J Med Genet 1991, 41:313-318

40. Folkerth RD, Alroy J, Lomakina I, Skutelsky E, Raghavan SS, Kolodny EH: Mucolipidosis IV: morphology and histochemistry of an autopsy case. J Neuropathol Exp Neurol 1995, 54:154-164

41. Tellez-Nagel I, Rapin I, Iwamoto T, Johnson AB, Norton WT, Nitowsky H: Mucolipidosis IV: clinical, ultrastructural, histochemical, and chemical studies of a case, including a brain biopsy. Arch Neurol 1976, 33:828-835

42. Wu SM, Gao F, Pang JJ: Synaptic circuitry mediating light-evoked signals in dark-adapted mouse retina. Vision Res 2004, 44: $3277-3288$
43. Frei KP, Patronas NJ, Crutchfield KE, Altarescu G, Schiffmann R: Mucolipidosis type IV: characteristic MRI findings. Neurology 1998, $51: 565-569$

44. Young RW: The renewal of photoreceptor cell outer segments. J Cell Biol 1967, 33:61-72

45. Obata S, Usukura J: Morphogenesis of the photoreceptor outer segment during postnatal development in the mouse (BALB/c) retina. Cell Tissue Res 1992, 269:39-48

46. Shen D, Wang X, Li X, Zhang X, Yao Z, Dibble S, Dong XP, Yu T, Lieberman AP, Showalter HD, Xu H: Lipid storage disorders block lysosomal trafficking by inhibiting a TRP channel and lysosomal calcium release. Nat Commun 2012, 3:731

47. Thompson EG, Schaheen L, Dang H, Fares H: Lysosomal trafficking functions of mucolipin-1 in murine macrophages. BMC Cell Biol 2007, 8:54

48. Reme CE, Wolfrum U, Imsand C, Hafezi F, Williams TP: Photoreceptor autophagy: effects of light history on number and opsin content of degradative vacuoles. Invest Ophthalmol Vis Sci 1999, 40:2398-2404

49. Kunchithapautham K, Coughlin B, Lemasters JJ, Rohrer B: Differential effects of rapamycin on rods and cones during light-induced stress in albino mice. Invest Ophthalmol Vis Sci 2011, 52:2967-2975

50. Kiselyov K, Jennigs JJ Jr, Rbaibi Y, Chu CT: Autophagy, mitochondria and cell death in lysosomal storage diseases. Autophagy 2007, 3:259-262

51. Clarke LA, Russell CS, Pownall S, Warrington CL, Borowski A, Dimmick JE, Toone J, Jirik FR: Murine mucopolysaccharidosis type I: targeted disruption of the murine alpha-L-iduronidase gene. Hum Mol Genet 1997, 6:503-511

52. Evers M, Saftig P, Schmidt P, Hafner A, McLoghlin DB, Schmahl W, Hess B, von Figura K, Peters C: Targeted disruption of the arylsulfatase $\mathrm{B}$ gene results in mice resembling the phenotype of mucopolysaccharidosis VI. Proc Natl Acad Sci U S A 1996, 93: 8214-8219

53. Hahn CN, del Pilar Martin M, Schroder M, Vanier MT, Hara Y, Suzuki K, d'Azzo A: Generalized CNS disease and massive GM1-ganglioside accumulation in mice defective in lysosomal acid beta-galactosidase. Hum Mol Genet 1997, 6:205-211 